\title{
Dielectric Elastomer Generators for Foot Plantar Pressure Based Energy Scavenging
}

\author{
Vishwa Goudar and Miodrag Potkonjak \\ Computer Science Department \\ University of California, Los Angeles \\ Los Angeles, USA
}

\begin{abstract}
Parasitic energy scavenging from human-generated vibrations with piezoelectric materials has long been studied in contrast to electromagnetic or conventional electrostatic transducers. Dielectric Elastomers (DEs) are now gaining notice as low-cost electrostatic transducers with high energy densities. However, their transduction mechanism is more intricate. DE Generators (DEGs) are functionally variable capacitors, which require fine-grained control of their charging cycles in order to maximize the energy transduced. Based on a detailed DEG model that incorporates an effective method to time the charge cycles, we contrast the energy scavenged from shoe strikes by DEGs that are virtually embedded into the shoe sole, to similar piezoelectric generators. This comparison for a plantar pressure dataset of a walking subject demonstrates a multiple order-ofmagnitude improvement in harvested energy.
\end{abstract}

\section{INTRODUCTION}

Rapid advances in miniaturization and communication technology has brought electronics to every aspect of human life. Portable electronic devices are ubiquitous, spanning communication devices, personal electronics, medical electronics, wearable systems and portable military devices. While major strides are being made in low-power electronics and wireless standards, battery energy densities have improved marginally [1]. Advances in power control, energy budgeting techniques and user education do provide a means to limit consumption rates and therefore battery size and the time between recharges[2] [3] [4]. Nevertheless, truly enhanced battery lifetimes and/or self-sustenance remain an elusive challenge that miniaturized energy harvesters aim to help overcome.

In furtherance of this vision, we study the transduction of the energy expended by humans during foot strikes. A variety of transduction mechanisms and materials have been identified and tested for human vibration harvesting [1]. However, in addition to performance, unobtrusiveness is key to usability and therefore to widespread adoption. This translates to a need for high energy-density harvesters. DEs are a promising new class of low-cost materials capable of 5 to 40 times the energydensity of piezoelectrics [5]. Like piezoelectrics, they also possess the ability to behave as energy transducers, actuators or sensors.

This work was supported in part by the NSF under awards CNS-0958369, CNS-1059435, and CCF-0926127, and is based upon research performed in collaborative facilities renovated with funds from the National Science Foundation under Grant No. 0963183, an award funded under the American Recovery and Reinvestment Act of 2009 (ARRA).
On the other hand, they are electrostatic transducers that use input mechanical work against the electrostatic forces capacitively stored by them, to vary their capacitance and achieve transduction. This means that, the DE "capacitor" must be charged before the mechanical work may be transduced, with the timing of the charge being key to transduction efficiency. For our application, this translates to charging the DE harvester at the moment when the plantar pressure is maximum. We propose an offline method to estimate the optimum chargetiming, apply a detailed DEG model that considers various electro-mechanical losses under a realistic transducer structure, and substantiate the proposed method with cross-validation tests on a plantar pressure dataset. Our results show significant improvements over piezoelectrics.

\section{RELATED WORK}

Miniaturized energy transduction mechanisms may be classified into ambient radiation, electromagnetic, electrostatic and piezoelectric, among others [6]. Whereas solar radiation transducers offer the most mature and impactful solutions, their placement requirements and the variable availability of light hampers adoptability. Electromagnetic generators are most effective and widely popular at the macro-scale, however, they are extremely obtrusive and difficult to miniaturize. Other mechanisms are limited in their output capacity $(\mu \mathrm{W}$ to low $\mathrm{mW}$ from human vibrations [1]). Piezoelectrics have higher energy densities as compared to conventional electrostatic and electromagnetic transducers, and are also well suited for harvesting human vibrational forces [6].

Reviews of human powered transduction spans heat transfer from the skin, vibration from foot strikes and knee joint movement, among others [1] [6]. A wide array of human motion has been proposed for exploitation including cranking, shaking, pushing, pumping, pulling as well as the isometric forces of squeezing and pushing [1] [7]. Human gait offers easy pickings as the most innocuous source of human power for transduction, resulting in a host of harvesting designs and related patents [8] that span various transduction mechanisms, transducer designs with varying levels of obtrusiveness and a wide range of energy output $(10 \mathrm{~mW}$ to $675 \mathrm{~mW})$.

DEGs are a relatively new entrant to the class of miniaturized generators. Although they are electrostatic transducers, their material properties distinguish them from conventional transducers in this class. An excellent survey of their 


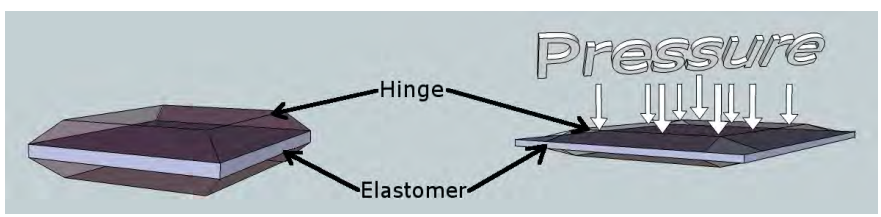

Fig. 1: Uniform biaxial stretch transducer configuration for dielectric elastomer generators.

transduction-related material properties, transducer configurations, recent applications, as well as, operational boundaries and lifetime issues are detailed in [5]. While DEGs require charging at high voltage so they may achieve their output potential, self-priming circuits have been proposed to overcome this [9]. Owing to their superior properties, researchers have proposed novel transducer configurations to retrofit DEGs to a host of applications initially proposed for the current bestof-breed, piezoelectrics.

\section{Dielectric Elastomer Generators}

\section{A. Material Properties and Transducer Configuration}

DEs are deformable yet incompressible insulating polymer films that can be built from a variety of materials. The most commonly used materials are acrylics and silicones due to their high electric permittivity $\left(\varepsilon_{r}\right)$, elasticity and relatively low mechanical and electrical losses. With electric permittivities 3 to 10 times that of air-gap electrostatic transducers, DEs can store and transduce more energy. They can be quite soft compared to piezoelectrics and their operational boundaries usually change when stretched, entertaining the possibility of extracting more energy from the film when it is stretched. DEs also exhibit non-linear stiffness behavior and can be viscoelastic, depending on the composite material.

Their relatively high elastic energy density means that the same amount (mass and volume) of material can store more energy, yielding less obtrusive and more productive transducers [5]. As highly elastic materials, they are also well suited for direct transduction and do not need an inertial mass akin to most electrostatic transducers, which not only lowers mechanical complexity, but also makes them highly efficient despite unpredictable driving forces at low frequencies, both of which are characteristic of human motion.

\section{B. Transduction Mechanism}

Fig. 1 shows the uniform biaxial transducer configuration that we propose for our application. It allows for a uniform deformation in the $\mathrm{x}$ and $\mathrm{y}$ directions when pressure is applied, with a proportionate reduction in material thickness (zdirection). In order to transduce mechanical energy, electrical charge must be added to the elastomer surface when it is stretched. On release of the mechanical pressure, the elastic forces in the DEG relax and are converted into electrostatic force. This may be conceptualized as an increase in electrical energy in the film when it relaxes, as like charges on the same surface are brought close together, and opposite charges are drawn apart. The conversion continues until the material completely relaxes, or, the increased electrostatic forces are able

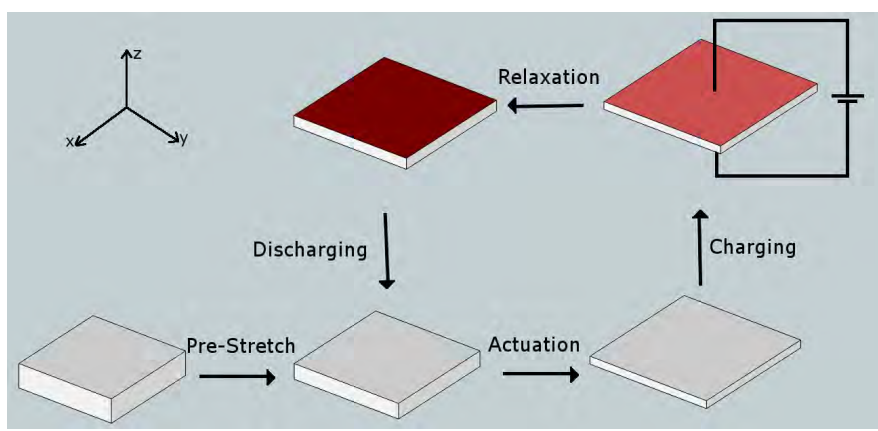

Fig. 2: Dielectric elastomer generators transduction cycle.

to maintain the film in a lower-stretch state. This transduction cycle is shown in Fig. 2.

Equations (1a) thru (1f) underscore the reason behind the high energy density of DEGs. If the length and width each increase by a factor of $\lambda$ when a DEG is stretched (uniform biaxial stretch), its incompressibility leads to a $\lambda^{2}$ factor decrease in thickness. So, if most of the charge on the film is conserved as it relaxes, at the end of relaxation, the electrical energy in the film will have increased by a factor of about $\lambda^{4}$. This is in contrast to a maximum $\lambda^{2}$ factor increase in energy in conventional electrostatic transducers, a limitation of their rigid structure. When combined with their high resistive and permittivity properties, DEs make for remarkable electrostatic transducers.

$$
\begin{aligned}
E & =\frac{Q^{2}}{2 C} \\
C_{0} & =\frac{\varepsilon_{0} \varepsilon_{r} A}{d} \\
C_{\lambda} & =\frac{\varepsilon_{0} \varepsilon_{r} \lambda^{2} A}{\frac{d}{\lambda^{2}}} \\
& =\lambda^{4} C_{0} \\
\Delta E_{D E G} & =\frac{Q^{2}}{2 C_{0}}\left(\frac{1}{\lambda_{\text {final }}^{4}}-\frac{1}{\lambda_{\text {init }}^{4}}\right) \\
& =\frac{C_{\lambda_{\text {final }}} V^{2}}{2}\left(\frac{\lambda_{\text {init }}^{4}}{\lambda_{\text {final }}^{4}}-1\right)
\end{aligned}
$$

\section{Transduction Model}

We now outline the detailed model, derived in [10], that we apply to compute the energy harvested by our proposed shoe strike energy harvesters. Each harvester is $9.5 \mathrm{~mm}$ by $9.5 \mathrm{~mm}$ in area with a single DE layer that is $1 \mathrm{~mm}$ thick. The harvesters are made of a VBH4910 acrylic DE, manufactured by $3 \mathrm{M}$. Table I lists the properties for this material [11] and its associated compliant electrode, as well as for the Suasis P-4 PZT [12], a piezoelectric transducer to which we will compare our DEG's performance.

The net energy harvested depends on the net electrical energy generated by the DE and the electrical and mechanical losses of the material (2). We ignore the negligible losses due to thermal stress. However, the electrical and mechanical losses, as well as the capacitance at various applied pressures, 


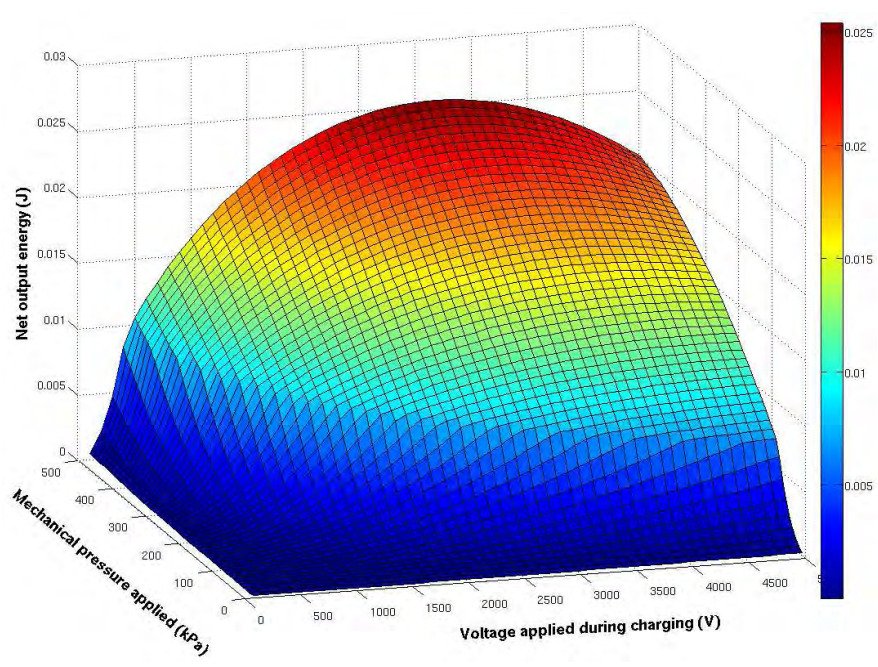

Fig. 3: Net Energy Scavenged by a single DEG as a function of applied mechanical pressure and voltage.

depend on the stress-stretch relationship of the material.

$$
E_{\text {harv }}=\Delta E_{D E G}-E_{\text {loss }_{\text {elec }}}-E_{\text {loss }_{\text {mech }}}
$$

A pressure $p$ applied by the foot in the z-direction is translated to an equibiaxial elastic stress in the $\mathrm{x}$ and $\mathrm{y}$-directions based on the transducer configuration (1). Mathematically, the resulting nominal elastic stress on the DEG film, $\sigma_{i}$, is expressed as a function of $p$ as follows.

$$
\sigma_{i}= \begin{cases}\frac{p x y}{z(2 x+2 y)} & \text { if } \mathrm{i}=\mathrm{x}, \mathrm{y} \\ 0 & \text { if } \mathrm{i}=\mathrm{z}\end{cases}
$$

Here $x, y$ and $z$ are the length, width and thickness, respectively. The amount of initial stretch, $\lambda_{\text {init }}$, of the VHB4910 material under this nominal stress is modeled with a MooneyRivlin strain energy function [13] that captures the nonlinearity therein, and was validated in [10]. It encompasses viscoelastic behavior to account for transient mechanical losses $E_{l_{\text {loss }} \text { mech }}$. With this model, we also account for a $200 \%$ prestretch in the $x$ and $y$ directions (Fig. 2), as this has been observed to improve energy outputs.

To compute the final stretch, $\lambda_{\text {final }}$, to which a charged DEG relaxes when the mechanical pressure is released, we must solve a force balance equation (3). The force balance equation expresses the net force that leads to a change in stretch, in terms of the mass and acceleration during this change (LHS of (3)). This force is achieved as a balance between the elastic forces (first term of (3)) and the sum of the electrostatic forces (second term of (3)) and gravitational forces (third term of (3)) acting on the DEG.

$$
\begin{aligned}
m x \lambda_{p} \frac{\partial^{2} \lambda}{\partial t^{2}}=y z & {\left[-3 \tilde{C}_{10}^{R}\left(2 \lambda_{p} \lambda-\frac{1}{\lambda_{p}^{3} \lambda^{3}}\right)\right.} \\
& \left.-3 \tilde{C}_{20}^{R}\left(2 \lambda_{p} \lambda-\frac{2}{\lambda_{p}^{3} \lambda^{3}}\right)-T_{p}\right] \\
& +\frac{z}{x^{2} y} \frac{1}{\lambda_{p}^{5} \lambda^{5}} \frac{Q^{2}}{\varepsilon_{0} \varepsilon_{r}}+\frac{z}{x \lambda_{p}^{2} \lambda^{2}} m g
\end{aligned}
$$

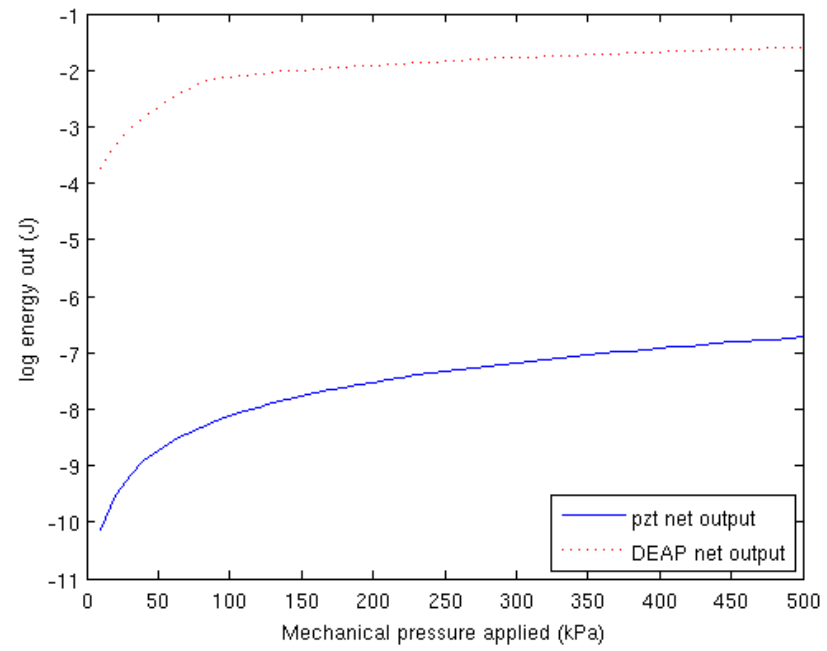

Fig. 4: Net Energy output (log scale) of a single VHB4910 DEG vs. PZT generator for different applied pressures.

Here, $m$ is the mass of the DEG, $\lambda_{p}$ is the pre-stretch and $T_{p}$ is the true elastic stress retained in the material due to its prestretched state (obtained from the Mooney-Rivlin function). $\tilde{C}_{i j}^{R}$ are terms of the Mooney-Rivlin model that capture the hyper-elastic and viscoelastic properties of the material. This differential equation is solved by a RungeKutta fourth-order method to arrive at $\lambda_{\text {final }}$.

Finally, electric losses of the DEG are modeled in terms of bulk and surface resistive losses (4). We also model resistive losses due to the compliant electrode, assuming a 1micron layer over the entire DEG surface area, and a resistive loss over a $1 \mathrm{k} \Omega$ resistor as part of the charging $\mathrm{RC}$ circuit.

$$
P_{\text {cond }_{B}}=\frac{V^{2}}{R_{b}} \quad P_{\text {cond }_{S}}=R_{s} I_{s}^{2}
$$

TABLE I: Material Properties for 3M VHB4910.

\begin{tabular}{c|c}
\hline \hline MATERIAL PROPERTY & VALUE \\
\hline \hline Dielectric Const. & 4.5 \\
Bulk Resistivity & $3.1 \times 10^{15} \Omega . \mathrm{cm}$ \\
Surface Resistance & $1.0 \times 10^{16} \Omega . \mathrm{cm} / \mathrm{m}^{2}$ \\
Density & $960 \mathrm{~kg} / \mathrm{m}^{3}$ \\
\hline Bulk Resistivity (carbon grease) & $1.17 \Omega . \mathrm{cm}$ \\
\hline Coupling Coeff. (PZT) & 0.66 \\
Dielectric Const. (PZT) & 1200 \\
Youngs Modulus (PZT) & $131 \times 10^{9} \mathrm{~Pa}$ \\
\hline \hline
\end{tabular}

\section{Optimum Charge-Timing Estimation}

A key observation from (1a) thru (1f) is that charge must be applied to DEGs as close to maximum stretch as possible, if net energy output is to be maximized. The lesser the stretch at the time of charging, the smaller the change in $\lambda$ between the time at which the film is charged and the time when it has relaxed, producing smaller changes in capacitance and electrical energy (1f). This is captured in the model by computing $\lambda_{\text {init }}$ at the instant when charge is expected to be applied.

To estimate the optimum charge-timing at each DEG in the shoe sole, we generate a likelihood distribution of the instants, 


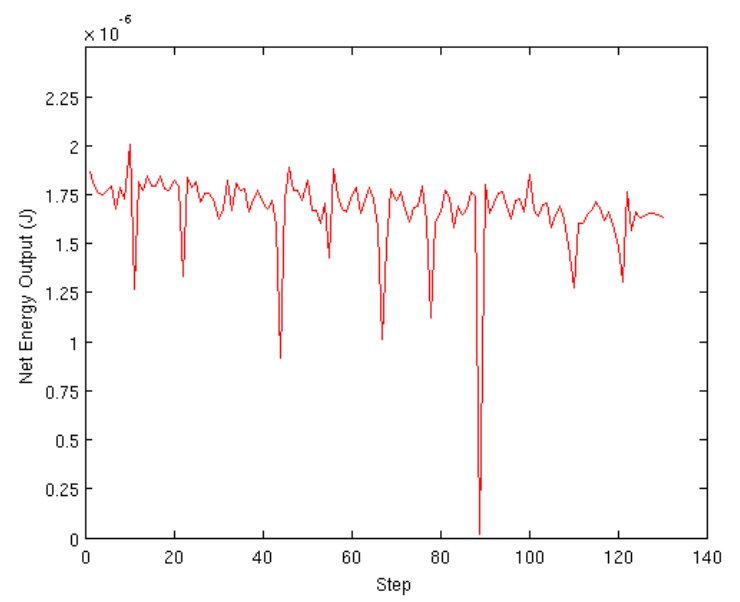

Fig. 5: Energy Scavenged by PZT generator array over 130 steps.

over a single step, when maximum pressure is observed at its location. This is achieved through a training dataset of pressure measurements over the sole, for a few steps taken by the subject. Optimum charge-timing at each DEG is then estimated at the mode of its likelihood distribution. A method with better net performance is proposed in [14], under the assumption that the shoe is also equipped with pressure sensors, resulting in a side-effect of increased cost.

\section{RESULTS}

Fig. 3 illustrates the energy output over a range of charging voltages and applied mechanical pressures when the DEG is charged. The pressure range shown is typical of foot strikes while walking. Our model shows that higher pressures yield more energy until the operational boundary is reached. However, higher voltages do not necessarily yield more energy. The energy gain tops out at an input voltage that is specific to the applied pressure. In Fig. 4, we compare the net energy output of one of our modeled DEGs to a $5 \mathrm{~mm}$ thick PZT transducer of the same dimensions over a range of input pressures. For this, we use the PZT transduction model from [15]. With energy plotted on a log scale, we see that DEGs are capable of energy outputs that are orders of magnitude higher than that of PZTs. However, we note that this may not be the ideal transducer configuration for PZTs. Next, we evaluate energy harvested from shoe-strikes based on a dataset of plantar pressure readings taken with the HERMES smart shoe [16]. The Hermes platform measures foot plantar pressure via a multi-sensory array of 99 sensors distributed uniformly across the shoe sole. Therefore, we assume placement of DEGs at these same locations on the shoe sole. Furthermore, for the range of maximum pressures seen over all steps in the dataset, we presume that each DEG is charged at $2.5 \mathrm{kV}$.

Fig. 5 shows the energy harvested by a $5 \mathrm{~mm}$ thick PZT generator for each step in the dataset, based on the total force observed over the entire sole, thereby modeling a more supportive PZT transducer configuration. In contrast, Fig. 6 exposes the energy harvested by the DEGs, as a sum over the energy generated by each of the 99 DEGs. Here, a distinction

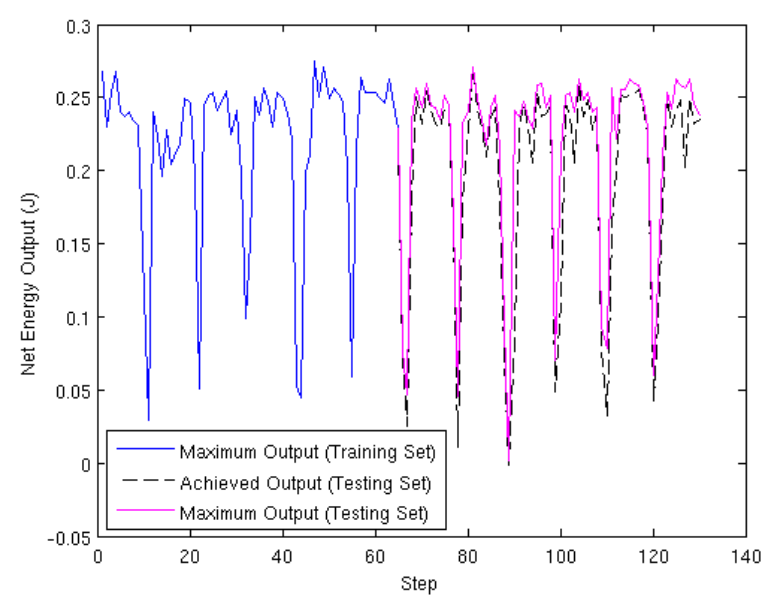

Fig. 6: Net Energy output by VHB4910 DEG array for trained and tested steps.

is made between the net energy output over the testing and training sets. Furthermore, for the training set, a comparison between the optimum charge-timing and our modal chargetiming technique reveals that the energy harvested is, on average, $85 \%$ of optimum. Finally, we see a 5 orders-ofmagnitude improvement in harvested energy by the DEGs, despite various mechanical and electrical losses, over the PZT generator.

\section{REFERENCES}

[1] J. A. Paradiso and T. Starner, "Energy scavenging for mobile and wireless electronics," IEEE Pervasive Computing, vol. 4, no. 1, pp. 18 27, Jan. 2005

[2] J. B. Wendt and M. Potkonjak, "Medical diagnostic-based sensor selection," in Sensors, 2011 IEEE, oct. 2011, pp. $1507-1510$.

[3] S. Meguerdichian et al., "Semantic multimodal compression for wearable sensing systems," in Sensors, 2010 IEEE, 2010, pp. 1149-1453.

[4] V. Goudar et al., "Power constrained sensor sample selection for improved form factor and lifetime in localized bans," in Wireless Health, 2012 ACM, 2012.

[5] R. D. Kornbluh et al., "From boots to buoys: promises and challenges of dielectric elastomer energy harvesting," Polymer, vol. 7976, no. 1, pp. 797 605-797 605-19, 2011.

[6] P. Mitcheson et al., "Energy harvesting from human and machine motion for wireless electronic devices," Proceedings of the IEEE, vol. 96, no. 9, pp. $1457-1486$, sept. 2008.

[7] M. Rofouei et al., "Optimization intensive energy harvesting," in Date, March 2012, pp. 272-275.

[8] J. Paradiso, "Systems for human-powered mobile computing," in Design Automation Conference, 2006 43rd ACM/IEEE, 2006, pp. 645 -650.

[9] T. McKay et al., "Self-priming dielectric elastomer generators," Smart Materials and Structures, vol. 19, no. 5, p. 055025, 2010.

[10] C. Jean-Mistral et al., "Modelling of dielectric polymers for energy scavenging applications," Smart Materials and Structures, vol. 19, no. 10 , p. $105006,2010$.

[11] “3M VHB ${ }^{\mathrm{TM}}$ tapes technical data sheet," 3M, St. Paul, MN.

[12] "Underwater silk road piezoelectric material product catalog," Suasis Underwater Systems, Kocaeli, Turkey.

[13] C. Jean-Mistral et al., "Dielectric polymer: scavenging energy from human motion," Proc of SPIE, vol. 6927, pp. 692711-692716, 2008.

[14] J. B. Wendt et al., "Spatiotemporal assignment of energy harvesters on a self-sustaining medical shoe," in Sensors, 2012 IEEE, oct. 2012.

[15] T. Wacharasindhu and J. W. Kwon, "A micromachined energy harvester from a keyboard using combined electromagnetic and piezoelectric conversion," Journal of Micromechanics and Microengineering, vol. 18, no. 10, p. 104016, 2008.

[16] H. Noshadi et al., "HERMES - mobile balance and instability assessment system,” in BIOSIGNALS, 2010, pp. 264-270. 